\title{
Synthesis and Characterization of Samarium or Lanthanum-Based Metal-Organic Frameworks with Crysophenine Ligand
}

\author{
Riska Yulianiza ${ }^{1}$, Agustino Zulys ${ }^{2}$, Jarnuzi Gunlazuardi ${ }^{3}$ \\ \{riska.yulianiza71@ui.ac.id ${ }^{1}$, zulys@ @sci.ui.ac.id ${ }^{2}$, jarnuzi@ui.ac.id ${ }^{3}$ \} \\ Department of Chemistry, Faculty of Mathematics and Natural Sciences, Universitas Indonesia, \\ Depok, West Jawa, Indonesia $16424^{1,2,3}$
}

\begin{abstract}
Lanthanide (Ln) Metal Organic Frameworks (Ln: Sm or La-MOF) have been synthesized with chrysophenine ligand $(\mathrm{CH})$ by solvothermal method. Furthermore, vibration spectroscopy, crystallography, morphology, and bandgap value of MOFs have also been studied. The FTIR result for Sm-MOF showed absorption peak shift of $\mathrm{C}=\mathrm{O}$ stretching mode in DMF to the lower wavenumber means Sm-MOF were successfully formed and the FTIR result of La-MOF showed a sharp peak at $1571.27 \mathrm{~cm}^{-1}$ which confirmed the success in formation of La-MOF. XRD results for both of the MOF showed crystalline characteristic. Still, for La-MOF indicated higher crystallinity, it revealed from SEM/EDS result which showed uniform arrangement between La and chrysophenine. EDS data proved the composition of $\mathrm{La}, \mathrm{O}, \mathrm{C}$, and $\mathrm{S}$ from chrysophenine in La-MOF. UV-DRS data showed the bandgap for Sm-MOF was $2.09 \mathrm{eV}$ at $590 \mathrm{~nm}$ and La-MOF was $1.98 \mathrm{eV}$ at $623 \mathrm{~nm}$ which means Ln-MOFs can be used as photocatalyst material under visible region.
\end{abstract}

Keywords: MOFs, Chrysophenine, Photocatalyst

\section{Introduction}

The increment of the human population on earth will cause much energy to help their activities like fossil fuel for transportation. However, this kind of fuel has been predicted will be used only until 2050 [1]. Therefore, scientists already start to find alternative energy. One of the efforts is utilizing sunlight as an energy source, which can change water into hydrogen and oxygen gas (water splitting). Hydrogen gas can be alternative energy because it's environmentally friendly and renewable [2]. Photocatalysis is one of the approaches to doing that process and utilizing sunlight as the source of energy [3].

Photocatalysis was first introduced by Fujishima and Honda in 1972 using $\mathrm{TiO}_{2}$ as the electrode under $\mathrm{UV}$ light [4]. However, bandgap of $\mathrm{TiO}_{2}$ is high for this process $(3.2 \mathrm{eV}$ for anatase phase and $3.0 \mathrm{eV}$ for rutile phase) and because of that only UV light can be absorbed (the percentage of it less than $5 \%$ in sunlight). Simultaneously, photocatalysis in water splitting is expected to work under visible light because the percentage of it is $40 \%$ in sunlight. Moreover, $\mathrm{Fe}_{2} \mathrm{O}_{3}$ and $\mathrm{BiVO}_{4}$ can be used as the electrode for the photocatalysis process. $\mathrm{Fe}_{2} \mathrm{O}_{3}$ is an earth-abundant, stable, and non-toxic material with an ideal bandgap (1.9-2.2 eV), but it has low mobility characteristics and slows at oxidation because of high surface area. Meanwhile, 
$\mathrm{BiVO}_{4}$ as photocatalyst material with bandgap $2.4 \mathrm{eV}$ and conduction band near $0 \mathrm{~V}_{\mathrm{RHE}}$ looked promising but need external bias for reduction and oxidation [5]. Therefore, research by scientists to find suitable photocatalyst material for water splitting in the visible region should be done to get alternative energy for our earth.

Scientists start to find suitable photocatalyst material by using Metal-Organic Frameworks (MOF). MOFs are a type of micropore crystalline material consisting of metal ions (or cluster) coordinated with organic molecule multidentate. MOFs have two essential parts, they are metal cation/cluster (it could be transition metals, $3 \mathrm{p}$ metals, or lanthanides) and organic linker (two carboxylates or more-, pyridyl-, or isolate functional group as cluster linker) [6]. MOFs have some advantages like a high volume ratio to the surface area, flexible design. They can also be used in visible regions and tunable porosity for hydrogen gas production in the water splitting process [4].

Water splitting will occur when the applied potential is $1.23 \mathrm{~V}$ at $\mathrm{pH}=0$ per 1 electron. Therefore, suitable photocatalyst material for this process should have a bandgap of more than $1.23 \mathrm{~V}$ or around $2.0 \mathrm{~V}$, which can work under the visible region $(\lambda=400-780 \mathrm{~nm})$. To achieve this goal, scientists have to consider metal (active site) in MOFS for water splitting. Metals from lanthanide like Samarium (Sm) and Lanthanum (La) can be considered for MOFs because lanthanide ions exhibited high amount of coordination number and diverse networking, which could create various and unpredicted structures of MOFs. Beside that, lanthanide ion as central ion in MOFs are widely adopted for tuning light absorption to ensure an efficient utilization of solar energy [7] MOFs based on lanthanide are very promising since organic linkers could act as an antenna that produces succesful photosensitization [8]. A photosensitizer like chrysophenine is an organic substance and organometal which can absorb and transfer visible light to the active site (metal) to become electron and make water reduction.

In this research, MOFs were made from samarium or lanthanum metal as the active site with chrysophenine as organic linker by solvothermal method at $170^{\circ} \mathrm{C}$ for $72 \mathrm{~h}$ with the usage of solvents like dimethylformamide (DMF) and water. Furthermore, the characterization of MOFs was done by FTIR, XRD, SEM-EDS and UV-Vis DRS.

\section{Materials and Methods}

All the chemicals used in this research were purchased from commercial sources and used without further purification. Multiple instruments such as Fourier Transform Infra-Red (FTIR) Prestige 21 Shimadzu, X-Ray Powder Diffraction (XRD), Shimadzu 6000, Scanning Electron Microscopy (SEM), and Diffuse Reflectance UV-Vis Shimadzu spectrophotometer 2200A were used for characterization of Ln-MOFs-CH.

\subsection{Synthesis of Lanthanide-MOFs (Sm-MOF and La-MOF)}

Ln-MOFs were synthesized by the solvothermal method by pouring each one mmol of $\mathrm{Sm}\left(\mathrm{NO}_{3}\right)_{3} \cdot 6 \mathrm{H}_{2} \mathrm{O}$ and $\mathrm{La}\left(\mathrm{NO}_{3}\right)_{3} \cdot 6 \mathrm{H}_{2} \mathrm{O}$ with one mmol chrysophenine in $4 \mathrm{~mL}$ dimethylformamide (DMF) and $11 \mathrm{~mL} \mathrm{H}_{2} \mathrm{O}$. Each system was stirred for 45 minutes and then moved to Teflon autoclave and heated for 72 hours at $170^{\circ} \mathrm{C}$. Furthermore, the crystals were washed using equates and $\mathrm{DMF}$, then dried in the oven at $60-80^{\circ} \mathrm{C}$ for 24 hours. 


\subsection{Characterization of Lanthanide-MOFs (Sm-MOF and La-MOF)}

Several instruments characterized the synthesized Ln-MOFs. FTIR was used to identify functional group and shift of absorption peak in MOFs with $\mathrm{KBr}$ as the pellet. XRD was used to check the crystallinity of MOFs. SEM was used to look at the morphology of MOFs and EDS to confirm the composition in MOFs. Furthermore, UV-Vis DRS was used to find the bandgap of MOFs formed.

\section{Results and Discussion}

Sm-MOF and La-MOF were synthesized by reacting each precursor $\mathrm{Sm}\left(\mathrm{NO}_{3}\right)_{3} \cdot 6 \mathrm{H}_{2} \mathrm{O}$ and $\mathrm{La}\left(\mathrm{NO}_{3}\right)_{3} \cdot 6 \mathrm{H}_{2} \mathrm{O}$ with chrysophenine ligand in DMF and water solvent. The polarity of the solvent used will control crystal growth [9]. DMF was used as the solvent because it has high polarity, good synthetic value until high-temperature, good thermal stability (even at the boiling point, $153^{\circ} \mathrm{C}$ ) and a wide range of solubility in the organic or inorganic compound [10]. The method used in this research was a solvothermal method, which is single crystal synthesis and other compounds that depend on mineral solubility in the solvent at a temperature higher than $100^{\circ} \mathrm{C}$, and it can form good quality MOF crystal for the structural characterization [9]. Characterization of synthesized MOFs were done by FTIR, XRD, SEM-EDS, and UV-DRS.

FTIR was used to identify the functional group in Sm-MOF and La-MOF at the range of wavenumber from 500 until $4000 \mathrm{~cm}^{-1}$. Fig. 1 (a) showed sharp peaks were confirmed the absortion peak shift from vibration $\mathrm{C}=\mathrm{O}$ stretching mode of $\mathrm{DMF}$ shifted to the lower wavenumber from 1671 to $1650.89 \mathrm{~cm}^{-1}$ shows the complex interaction between DMF and $\mathrm{Sm}$ MOF were successfully formed. The other is at $1601.12 \mathrm{~cm}^{-1}$ indicated that $\mathrm{C}=\mathrm{C}$ from chrysophenine ligand shifted to the higher wavenumber from 1597.81 to $1601.12 \mathrm{~cm}^{-1}$, also showing the interaction of $\mathrm{C}=\mathrm{C}$ with $\mathrm{Sm}^{3+}$ occur. Furthermore, peaks at $2981.15 \mathrm{~cm}^{-1}$ (sharp peak) and $3352.69 \mathrm{~cm}^{-1}$ (broad peak) showed the $\mathrm{O}-\mathrm{H}$ stretching mode from water molecules in Sm-MOF [8]. Fig. 1 (b) showed two sharp peaks at 1352.32, and $1402.08 \mathrm{~cm}^{-1}$ indicated both symmetry and asymmetric vibrations of $\mathrm{C}=\mathrm{O}$, respectively. Furthermore, a sharp peak at $1571.27 \mathrm{~cm}^{-1}$ was observed. It indicated the success of the complex interaction between $\mathrm{C}=\mathrm{O}$ from DMF with $\mathrm{La}^{3+}$ and absorption peak at $3455.53 \mathrm{~cm}^{-1}$ revealed $\mathrm{O}-\mathrm{H}$ functional group from water [8].

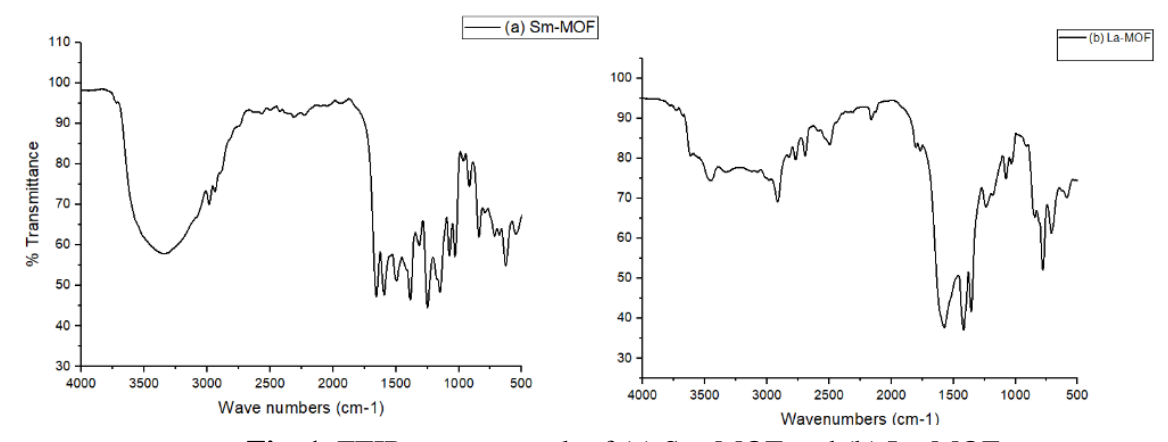

Fig. 1. FTIR spectra result of (a) Sm-MOF and (b) La-MOF 
The crystallinity of synthesized MOFs was then observed by using XRD. The result is SmMOF and La-MOF have crystallinity characteristics, but La-MOF showed higher crystallinity than Sm-MOF, as shown in Fig. 2. La-MOF has characteristic peaks at 16.5 and 33.38 while Sm-MOF at 4.16 and 8.32. Moreover, SEM-EDS is used to look at the morphology, and EDS identifies the MOF's composition. From SEM result showed that La-MOF has a uniform arrangement of the active site $\left(\mathrm{La}^{3+}\right)$. The EDS revealed $\mathrm{La}, \mathrm{O}, \mathrm{C}$, and $\mathrm{S}$ from the chrysophenine ligand as shown in Fig. 3 and 4.
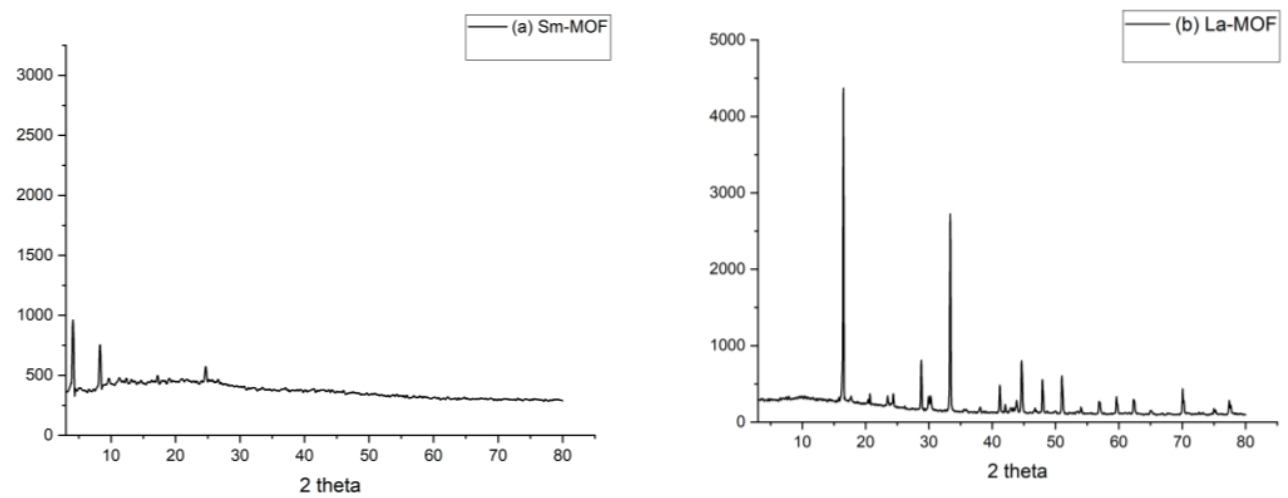

Fig. 2. XRD Pattern for (a) Sm-MOF and (b) La-MOF

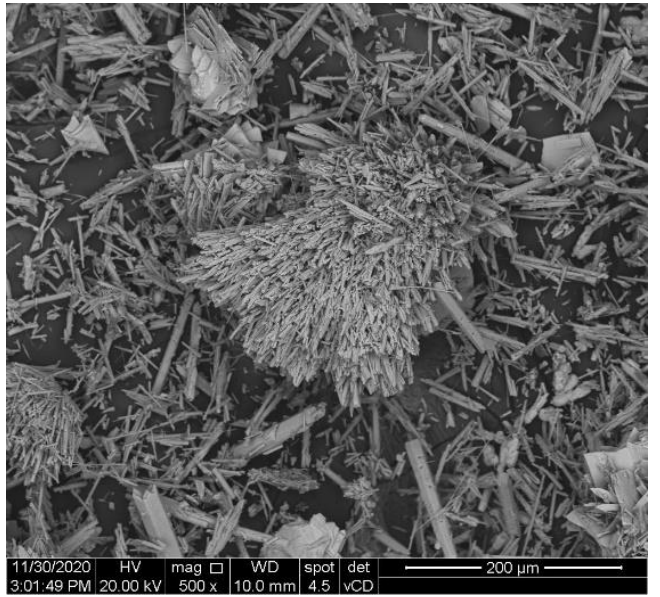

Fig. 3. SEM image of La-MOF with scale bar 200 $\mu \mathrm{m}$

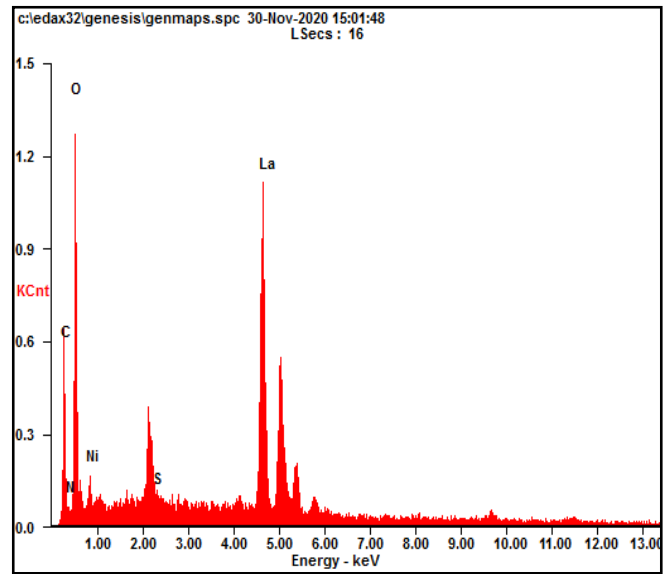

Fig. 4. EDS spectra of La-MOF 
Table 1. Composition in La-MOF

\begin{tabular}{ccc}
\hline Element & Weight (\%) & Atoms (\%) \\
\hline $\mathrm{C}$ & 10.98 & 31.36 \\
$\mathrm{~N}$ & 01.22 & 02.99 \\
$\mathrm{O}$ & 21.62 & 46.36 \\
$\mathrm{Ni}$ & 07.16 & 04.19 \\
$\mathrm{~S}$ & 00.64 & 00.69 \\
$\mathrm{La}$ & 58.38 & 14.42 \\
$\mathrm{C}$ & 10.98 & 31.36 \\
\hline
\end{tabular}

Measurement of bandgap value is one of the requirements to check whether the material can be utilized as a photocatalyst or not. Bandgap value means how much energy is required by photocatalyst for photocatalysis process and determines wavelength from the light source, whether in UV or visible light range. Characteristic of photocatalyst which can be used under visible light (400-700 $\mathrm{nm})$ is having bandgap range around 1.9-2.2 eV.
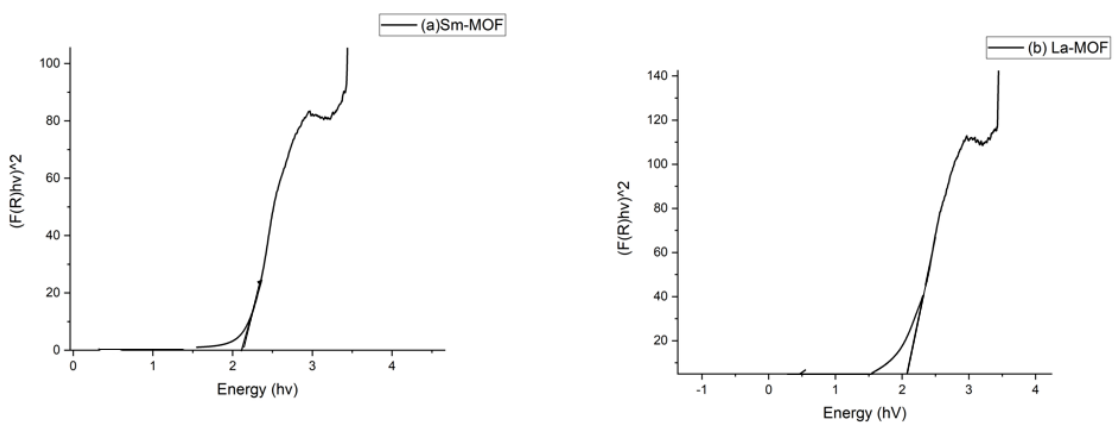

Fig. 3. Bandgap measurement of (a) Sm-MOF and (b)La-MOF

Measurement of the bandgap for both Sm-MOF and La-MOF are showed in Fig. 5 by using the Tauc plot, and the results of reflectance were converted to Kubelka-Munk function with curve $\mathrm{F}(\mathrm{R})$ as the result. UV-DRS data showed that the Sm-MOF bandgap was $2.09 \mathrm{eV}$ at 590 $\mathrm{nm}$ and La-MOF was $1.98 \mathrm{eV}$ at $623 \mathrm{~nm}$ which confirmed that Sm-MOF and La-MOF can be used as photocatalyst material under visible region.

\section{Conclusion}

In this research, Sm-MOF and La-MOF were successfully synthesized by using the solvothermal method. From FTIR result, there was absorption peak shift of DMF to the lower wavenumber from 1671 to 1650.89 in Sm-MOF indicated the ligation between DMF and SmMOF formed, and there was sharp peak observed at $1571.27 \mathrm{~cm}^{-1}$ in La-MOF indicated complex interaction between $\mathrm{La}^{3+}$ ions and $\mathrm{C}=\mathrm{O}$ formed. XRD result showed that La-MOF has higher crystallinity than Sm-MOF, it revealed from SEM-EDS result, which showed uniformly 
arrangement of La and chrysophenine as the ligand. Bandgap value of Sm-MOF and La-MOF were obtained from UV-DRS. Sm-MOF's bandgap value was $2.12 \mathrm{eV}$ at $585 \mathrm{~nm}$, and La-MOF was $2.07 \mathrm{eV}$ at $599 \mathrm{~nm}$. These data showed that Sm-MOF and La-MOF suitable as photocatalyst material under visible light (ideal bandgap 1,9-2,2 eV) for further application in the watersplitting process.

Acknowledgments. The authors would like to acknowledge the Directorate of Research and Community Engagements, Universitas Indonesia, to support this research through Hibah PUTI UI 2020. The authors also state that no conflict of interest in this research.

\section{References}

[1] J. Edmonds and J. Reilly, "Global energy production and use to the year 2050," Energy, vol. 8, no. 6, pp. 419-432, 1983, doi: 10.1016/0360-5442(83)90064-6.

[2] O. Bičáková and P. Straka, "Production of hydrogen from renewable resources and its effectiveness," Int. J. Hydrogen Energy, vol. 37, no. 16, pp. 11563-11578, 2012, doi: 10.1016/j.ijhydene.2012.05.047.

[3] Pankaj Chowdhury, "SOLAR AND VISIBLE LIGHT DRIVEN PHOTOCATALYSIS FOR SACRIFICIAL HYDROGEN GENERATION AND WATER DETOXIFICATION WITH CHEMICALLY MODIFIED TiO2," Appl. Sol. Visible Light Driven Photocatal., vol. 2, no. August, 2012.

[4] W. Wang, X. Xu, W. Zhou, and Z. Shao, "Recent Progress in Metal-Organic Frameworks for Applications in Electrocatalytic and Photocatalytic Water Splitting," 2017, doi: 10.1002/advs.201600371.

[5] S. Kumar, D. D. Rodene, and R. B. Gupta, "Recent advancements in semiconductor materials for photoelectrochemical water splitting for hydrogen production using visible light," Renew. Sustain. Energy Rev., vol. 89, no. March, pp. 228-248, 2018, doi: 10.1016/j.rser.2018.03.063.

[6] R. A. Patil, C. A. Weatherly, and D. W. Armstrong, Chiral Gas Chromatography, Second Edi. Elsevier B.V., 2018

[7] D. A. Wulandari, A. Zulys, and E. Kusrini, "Samarium Complexes from 2,6Naphtalenedicarboxylate: Synthesis, Photocatalytic Properties and Degradation of Methylene Blue," IOP Conf. Ser. Mater. Sci. Eng., vol. 546, no. 4, pp. 1-7, 2019, doi: 10.1088/1757899X/546/4/042050.

[8] I. O. P. C. Series and M. Science, "Synthesis, Structural, Spectroscopic, and Morphology of Metal-Organic Frameworks Based on La ( III ) and Ligand 2, 6-Napthalenedicarboxylic acid ( La-MOFs ) for Hydrogen Production Synthesis, Structural, Spectroscopic , and Morphology of Metal-Organ," no. Iii, pp. 0-7, 2019, doi: 10.1088/1757-899X/546/4/042005.

[9] R. Seetharaj, P. V Vandana, P. Arya, and S. Mathew, "Dependence of solvents, pH, molar ratio and temperature in tuning metal organic framework architecture," Arab. J. Chem., 2016, doi: 10.1016/j.arabjc.2016.01.003.

[10] B. I. Pastoriza-santos and L. M. Liz-marza, "FEATURE ARTICLE N, N -Dimethylformamide as a Reaction Medium for Metal Nanoparticle Synthesis," pp. 679-688, 2009, doi: 10.1002/adfm.200801566. 